\title{
CONTAMINATION PAR ANGUILLICOLA CRASSUS \\ DE CINQ POPULATIONS D'ANGUILLES (ANGUILLA ANGUILLA) \\ DU LITTORAL DE BRETAGNE SUD (FRANCE)
}

\author{
B. SAUVAGET (1), D. FATIN (2) ET C. BRIAND (2)
}

(1) La Gautrie, 44650 Corcoué sur Logne, France.

(2) Institution d'Aménagement de la Vilaine, Boulevard de Bretagne, 56130 la RocheBernard, France.

\section{RÉSUMÉ}

Les anguilles prélevées dans cinq zones de salinité variable du littoral de Bretagne sud, ont été examinées pour leur infestation présente et passée par le parasite Anguillicola crassus (Nematoda, dracunculoidea). Les anguilles vivant dans des milieux peu salés au contact de la zone dulçaquicole sont très contaminées avec des prévalences supérieures à $90 \%$, dont $61 \%$ concernent des prévalences présentes. Par contre, celles vivant dans des conditions estuariennes (Golfe du Morbihan) sont relativement épargnées avec des prévalences de l'ordre de $15 \%$, dont en moyenne moins de la moitié concernent des prévalences présentes. La salinité des baies et des estuaires semble donc pouvoir préserver de la contamination les populations d'anguilles côtières dont la gestion devient un enjeu prioritaire pour le stock d'anguilles.

Mots-clés : Anguillicola crassus, Anguilla anguilla, salinité, parasite.

\section{CONTAMINATION BY ANGUILLICOLA CRASSUS OF FIVE COASTAL EEL POPULATIONS (ANGUILLA ANGUILLA) IN BRITTANY (FRANCE)}

\begin{abstract}
Eels were sampled in five areas of variable salinity from the coast of south Brittany and examined for their past and present infection by the parasite Anguillicola crassus (Nematoda, dracunculoidea). Eels living in low salinity areas, in contact to the freshwater area, were highly contaminated with prevalence larger than $90 \%$, with $61 \%$ concerning present prevalence. In contrast, eels living in estuarine conditions (Golfe du Morbihan) were relatively preserved with prevalences close to $15 \%$, with less than the half concerning present prevalence. Therefore, the salinity of bays and estuaries seems to preserve from contamination the coastal eel populations whose management becomes a major goal for the eel stock.
\end{abstract}

Key-words : Anguillicola crassus, Anguilla anguilla, salinity, parasite.

\section{INTRODUCTION}

Les anguilles parasitées par Anguillicola crassus présentent une altération de la vessie natatoire plus ou moins marquée entraînant un affaiblissement général de 
l'individu (BLANC, 1994). Cette diminution des performances pourrait avoir des effets néfastes sur la migration transocéanique et la reproduction (LEFEBVRE et al., 2002a).

La transmission d'Anguillicola crassus au sein d'une population d'anguilles dépend de plusieurs facteurs : 1) la disponibilité en hôtes intermédiaires (KENNEDY et FITCH, 1990 ; BLANC, 1994) et en hôtes paraténiques (ASHWORTH et BLANC, 1997 ; MORAVEC et SKORIKOVA, 1998) ; 2) le temps passé dans un milieu contaminé et la quantité de nourriture ingérée (ADAM, 1997) ; 3) les températures extrêmes, néfastes au cycle parasitaire (DE CHARLEROY et al., 1989 ; KENNEDY et FITCH, 1990 ; BLANC, 1994 ; KIRK et al., 2000) ; 4) la salinité car elle a un effet négatif sur le cycle parasitaire (DE CHARLEROY et al., 1989 ; KENNEDY et FITCH, 1990 ; BLANC, 1994 ; KIRK, et al., 2000) et donc sur la prévalence du parasite (NIELSEN, 1997 ; LEFEBVRE et al., 2002a).

Toutefois, des travaux récents se basant sur des indices de dégradation de la vessie natatoire mettent en doute l'effet de la salinité, en montrant que des anguilles saines pouvaient avoir été parasitées auparavant et que la contamination passée est indépendante de la salinité du milieu (LEFEBVRE et al., 2002a, b). L'absence d'infestation parasitaire des stocks maritimes par le parasite Anguillicola crassus est un des éléments clé de la gestion de l'anguille. L'analyse des modalités de la contamination des anguilles côtières par Anguillicola crassus est donc un préalable à l'adoption de mesures préférentielles de gestion pour les stocks côtiers.

Le parasite Anguillicola crassus était présent uniquement sur le bassin versant de la Vilaine en 1991. En 1998, sa présence s'est étendue à tous les grands cours d'eau bretons (Aulne, Blavet, Vilaine) et 19 stations de pêche électrique sur les 27 du réseau hydrobiologique et piscicole breton sont contaminées (VOLPATO, 1998). L'objectif de cette étude est de présenter un instantané de la situation épidémiologique des anguilles des côtes sud de la Bretagne vis à vis d'Anguillicola crassus.

\section{MATÉRIEL ET MÉTHODE}

Les anguilles ont été collectées par pêche expérimentale au verveux dans trois stations de salinité variable (Tableau I) où la pêche professionnelle est absente : le marais de Fouesnant $(n=34,26$ avril 2001), le Ster de Lesconil ( $n=116,16$ mai 2001) et la rivière de Pont l'Abbé en amont ( $n=37,8$ juin 2001). Le marais de Fouesnant est peu saumâtre, il est relié à la mer par un chenal qui l'alimente uniquement lors des grandes marées. La station sur la rivière de Pont l'Abbé est dulçaquicole, elle est située en amont de la limite de salure des eaux. Le Ster de Lesconil est moyennement saumâtre à salé en raison du jeu des marées et des apports limités de son bassin versant en eau douce (Figure 1). Les salinités des trois sites ont été mesurées lors des campagnes de pêche.

Les anguilles sont également collectées auprès des pêcheurs professionnels sur le Golfe du Morbihan, en rivière d'Auray $(n=150)$ et à Truscat $(n=108)$. Les anguilles d'Auray ont été pêchées entre le 11 et le 19 juillet 2001, celles de Truscat entre le 30 juillet et le 4 août 2001. La salinité est mesurée à mi-profondeur à pleine mer lors de seize campagnes d'avril à novembre en 2001. La salinité moyenne est de 32,9 $(\min =25,7$; $\max =35)$ pour la rivière d'Auray et 33,2 pour Truscat $(\min =26$; $\max =35,2)$ (données IFREMER, La Trinité).

Toutes les anguilles disséquées sont au stade jaune, elles ont une taille moyenne par site variant entre $343 \mathrm{~mm}$ à Pont L'Abbé et $558 \mathrm{~mm}$ à Fouesnant. La vessie natatoire est extraite puis examinée à l'œil nu et sa dégradation est évaluée par trois critères qualitatifs notés de zéro à deux (LEFEBVRE et al., 2002b) : (1) l'opacification de la paroi de la vessie natatoire ; (2) la pigmentation externe du tissu conjonctif et la 
présence d'exudats remplaçant le gaz dans le lumen de la vessie natatoire ; (3) l'épaisseur de la paroi de la vessie natatoire. La contamination de la vessie natatoire est ensuite décrite par l'intensité moyenne (nombre moyen de parasites vivants par hôte infecté) et l'abondance moyenne (nombre moyen de parasites vivants par hôte examiné). L'intensité et l'abondance moyennes de parasites sont des descripteurs de l'infection présente. En l'absence de parasites, la dégradation de la vessie natatoire permet d'évaluer la contamination passée, c'est à dire la possibilité que l'anguille ait été en contact avec le parasite dans le passé. Cette contamination passée diffère de la prévalence, qui correspond à un instantané du pourcentage d'anguille contaminé, car les anguilles n'ont pas forcément été en présence du parasite au même moment.

\section{Tableau I}

Intensité et abondance moyennes du parasite Anguillicola crassus et salinité des zones échantillonnées.

\section{Table I}

Mean Intensity and abundance of the parasite Anguillicola crassus and salinity of the sampling areas.

\begin{tabular}{cccccc}
\hline Station & Pont l'Abbé & Fouesnant & Lesconil & Auray & Truscat \\
\hline Salinité $(\mathrm{g} / \mathrm{L})$ & 0 & $5(0-10)$ & $22(10-35)$ & $32,9(25-35)$ & $33,2(25-35)$ \\
\hline Intensité moyenne & 4 & 6 & 4 & 2 & 2 \\
$\mathrm{n}=$ & 25 & 21 & 6 & 16 & 1 \\
écart-type & 5 & 9 & 5 & 2 & $/$ \\
\hline Abondance moyenne & 3,03 & 3,82 & 0,19 & 0,25 & 0,02 \\
$\mathrm{n}=$ & 37 & 34 & 116 & 150 & 108 \\
écart-type & 4,86 & 7,69 & 1,34 & 0,94 & 0,19 \\
\hline
\end{tabular}
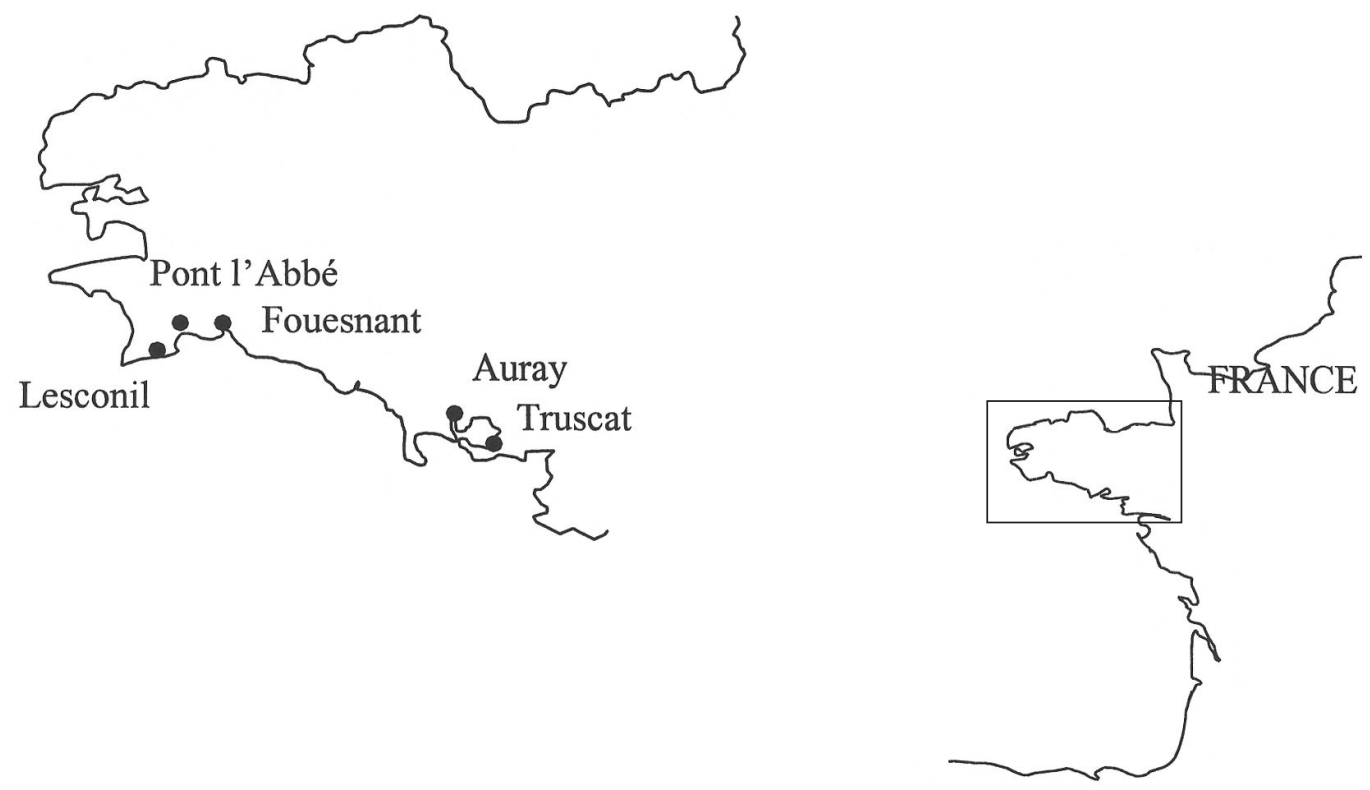

Figure 1:

Localisation des cinq populations d'anguilles étudiées en 2001.

Figure 1:

Localisation of the five eel populations studied in 2001. 


\section{RÉSULTATS}

Les stations de Pont l'Abbé et de Fouesnant sont fortement contaminées avec des prévalences de $67,6 \%(n=37)$ et $61,8 \%(n=34)$. Par contre, les stations de Lesconil et du Golfe du Morbihan sont peu contaminées (Lesconil : 5,2 \%, $n=116$; Auray : 10,7 \%, $\mathrm{n}=150$; Truscat : 0,9 \%, $\mathrm{n}=108$ ) (Figure 2). Les stations diffèrent entre elles en terme de prévalence (Test du $\chi^{2}(4)=557, p<0,001$ ) et d'abondance moyenne (Test de KruskalWallis, $H(4,445)=164,8, p<0,001)$. Par contre, l'intensité moyenne, qui compare les anguilles contaminées entre elles, ne diffère pas entre les sites $(H(4,69)=4,31, p=0,36)$ même si on écarte la station de Truscat de l'analyse car elle ne comporte qu'une anguille parasitée $(H(3,68)=4,22, p=0,24)$ (Tableau I). L'abondance est négativement corrélée avec la salinité ( $R$ Spearman $=-0,42, n=445, p<0,001$ ).

La contamination passée, calculée sur le nombre d'anguilles non contaminées lors de l'autopsie, est élevée pour les stations de Pont l'Abbé (75 \%, $n=12)$ et de Fouesnant $(84,6 \%, n=13)$ et faible pour les 3 autres stations (Lesconil : 11,8 \%, $n=110$; Auray : $8,2 \%, n=134$; Truscat : 11,2 \%, $n=107$ ) (Figure 2). Elle diffère entre les stations (Test du $\left.\chi^{2}(4)=541, p<0,001\right)$ et est négativement corrélée avec la salinité ( $R$ Spearman $=-0,19, \mathrm{n}=54, \mathrm{p}<0,05)$.

Quand on additionne la prévalence et la contamination passée, il apparaît que les anguilles des stations de Pont l'Abbé et de Fouesnant sont très contaminées (Pont l'Abbé amont : 91,9\%, n=37; Fouesnant : 94,1\%, n=34). A l'opposé, les anguilles des stations de Lesconil et du Golfe du Morbihan sont assez peu touchées (Lesconil : $16,4 \%, \mathrm{n}=116$; Auray : $18 \%, \mathrm{n}=150$; Truscat : $12 \%, \mathrm{n}=108$ ) (Figure 2).

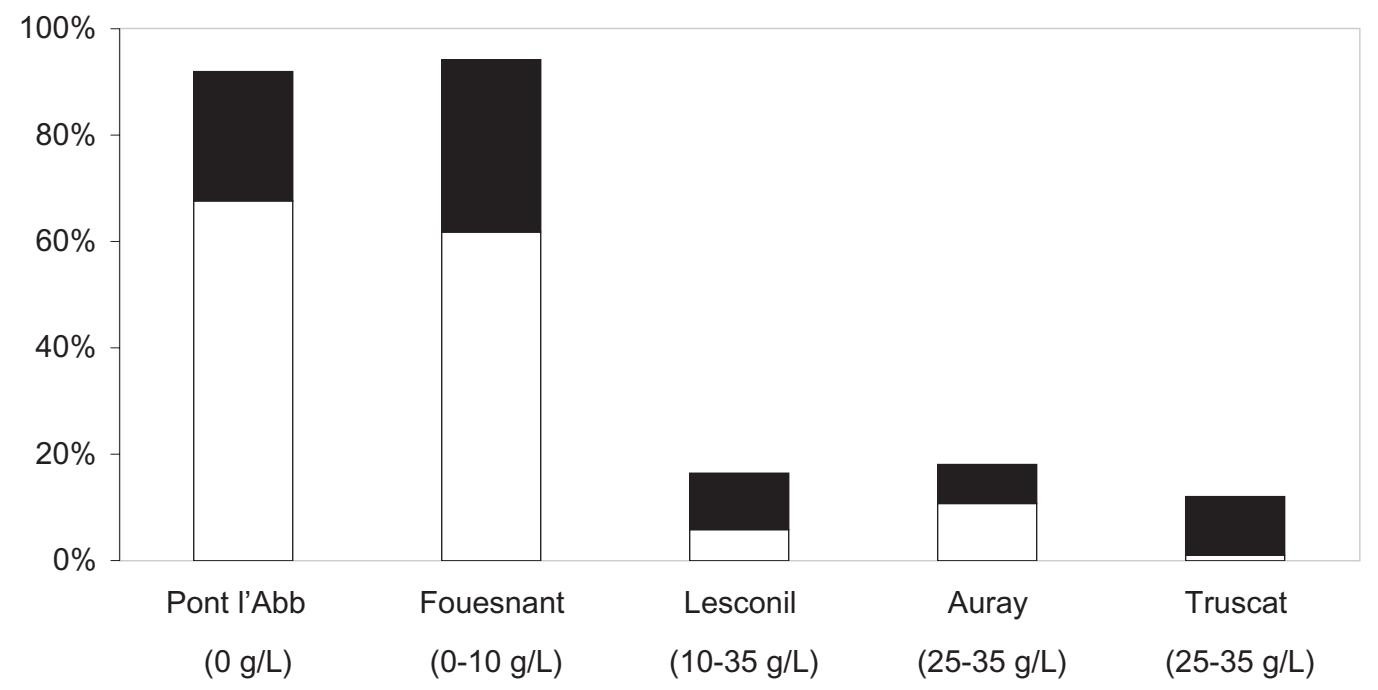

anguilles non contamin es avec vessies natatoires endommag es (contamination pass e)

$\square$ anguilles contamin es

\section{Figure 2}

Proportion d'infectation courante et passée des anguilles par Anguillicola crassus pour les cinq populations étudiées en 2001.

\section{Figure 2}

Proportion of current and past infections of eels by Anguillicola crassus for the five populations studied in 2001 


\section{DISCUSSION}

Les stations dont la salinité n'est pas assez forte pour être un obstacle à Anguillicola crassus sont fortement contaminées, c'est le cas de Fouesnant et de Pont l'Abbé. Par contre, celles dont la salinité est élevée sont relativement épargnées, c'est le cas de Lesconil et des stations du Golfe du Morbihan.

La station de Lesconil est peu touchée par Anguillicola crassus (16,4\%) malgré la faible distance qui sépare le site d'échantillonnage du bassin versant de faible superficie. De même, les anguilles du Golfe du Morbihan sont peu contaminées (Auray : $18 \%$, Truscat : $12 \%$ ) alors que des rivières se jettent à quelques kilomètres des sites d'échantillonnage. La faible prévalence observée sur ces stations souligne le caractère majoritairement sédentaire des anguilles qui y vivent.

La différence de contamination entre les deux stations du Golfe est peut-être liée à la distance de la source d'eau contaminée la plus proche. Les résultats vont dans ce sens car $10,7 \%$ des anguilles proches de la rivière d'Auray portent au moins un parasite vivant contre 0,9\% seulement pour celles de Truscat au sud-est du Golfe. Cependant, quatre anguilles de Truscat portent des restes de parasites ce qui signifie qu'elles ont été en contact avec un milieu contaminé.

La majorité des anguilles vivant dans des sites en eau saumâtre et salée ont une vessie natatoire indemne.

Des résultats obtenus en Camargue (LEFEBVRE et al., 2002a,b) montrent une forte contamination chez les anguilles argentées en milieu saumâtre mais pour des salinités plus faibles que celles rencontrées dans les sites bretons. II semble donc exister une barrière à la contamination par Anguillicola crassus pour les sites de forte salinité.

\section{CONCLUSION}

Le parasite Anguillicola crassus est très présent dans le sud de la Bretagne dès qu'il rencontre des conditions favorables à sa dissémination, c'est à dire dès que les anguilles sont au contact de l'eau douce. Dans le cas des marais côtiers, comme celui de Fouesnant, le maintien du caractère salé, par l'amélioration des échanges avec la mer, pourrait permettre de diminuer la contamination des anguilles. Dans le cas de baies et de la partie maritime des estuaires, les anguilles sont peu contaminées, et cette absence de contamination souligne l'importance de la préservation des stocks côtiers d'anguilles.

\section{REMERCIEMENTS}

Nous tenons à remercier les pêcheurs, José Gouyen, Serge Lefranc, Mikaël Corlay, Pascal Cadéro, Gérard Lebourvenec, les équipes de l'Ifremer la Trinité et de la Tour du Vallat, avec des remerciements particuliers à Isabelle Peronnet, Sophie Lemestre, Jean-Pierre Allenou, François Levebvre et Alain Crivelli pour leur assistance scientifique et technique.

\section{BIBLIOGRAPHIE}

ADAM G., 1997. L'anguille européenne (Anguilla anguilla L. 1758) : dynamique de la souspopulation du lac de Grand-Lieu en relation avec les facteurs environnementaux et anthropiques. Thèse, Université Toulouse III, $353 \mathrm{p}$.

ASHWORTH, S.T. et BLANC, G., 1997. Anguillicola crassus, un colonisateur agressif récemment introduit dans les stocks européens d'anguilles. Bull. Fr. Pêche Piscic., 344/345, 335-342. 
BLANC G., 1994. Biologie du cycle d'Anguillicola crassus (Nematoda, Dracunculoidea), contrôle thérapeutique de ses populations. Thèse, Université de Perpignan, Montpellier, $355 \mathrm{p}$.

DE CHARLEROY D., THOMAS K., BELPAIRE C. et OLLEVIER F., 1989. The viability of the free-living larvae of Anguillicola crassus. J. Appl. Ichthyol., 5, 154-156.

KENNEDY C. R. et FITCH D. J., 1990. Colonization, larval survival and epidemiology of the nematode Anguillicola crassus, parasitic in the eel, Anguilla anguilla, in Britain. J. Fish. Biol., 36, 117-131.

KIRK, R.S., KENNEDY, C.R. et LEWIS, J.W., 2000a. Effect of salinity on hatching, survival and infectivity of Anguillicola crassus (Nematoda: Dracunculoidea) larvae. Dis. Aquat. Org., 40, 211-218.

KIRK, R.S., LEWIS, J.W. et KENNEDY, C.R., 2000b. Survival and transmission of Anguillicola crassus Kuwahara, Niimi et Itagaki, 1974 (Nematoda) in seawater eels. Parasitology, 120, 289-295.

LEFEBVRE F., ACOU A., POIZAT G. et CRIVELLI A. J., 2002a. Anguicollosis among silver eels : a 4-year survey in 4 habitats from camargue (Rhône delta, South of France). Bull. Fr. Pêche Piscic., sous presse.

LEFEBVRE F., CONTOURNET P. et CRIVELLI A. J., 2002b. The health state of the eel swimbladder as a measure of parasite pressure by Anguillicola crassus. Parasitology, 124, 457-463.

MORAVEC, F. et SKORIKOVA, B., 1998. Amphibians and larvae of aquatic insects as new paratenic hosts of Anguillicola crassus (Nematoda: Dracunculoidea), a swimbladder parasite of eels. Dis. Aquat. Org., 34, 217-222.

NIELSEN, M. E., 1997. Infection status of the swimbladder worm, Anguillicola crassus in silver stage European eel, Anguilla anguilla, from three different habitats in Danish waters. J. Appl. Ichthyol., 13, 195-196.

VOLPATO P., 1998. Recherche du parasite Anguillicola crassus en région Bretagne. Conseil Supérieur de la Pêche, Rennes, 11 p. 